\title{
Malignant Melanoma of the Oral Cavity
}

\author{
- Report of a Case- \\ SHIGEJIRO KURITA, KAZUTO NAGATA AND SHIGENOBU MIHASHI \\ Department of Otolaryngology, Kurume University, School of Medicine, \\ Kurume, 830 Japan
}

Received for publication April 10, 1982

\begin{abstract}
Summary: Malignant melanoma of the oral cavity is a relatively rare condition. A 57-year-old female case of malignant melanoma arising from the oral cavity was treated in Kurume University Hospital. The initial symptom was a tumor formation of the neck. The primary tumor was located on the right upper gingiva. The patient was successfully treated with partial maxillectomy and radical neck dissection and is currently alive 2 years and 10 months after the initial treatment with no evidence of recurrent tumor.
\end{abstract}

Key words : malignant melanoma - melanoma - oral cavity - melanoma of oral cavity

\section{Introduction}

Malignant melanoma of the oral cavity is a rare condition. Generally speaking, prognosis of malignant melanoma of the mucous membrane is poorer than that of the skin. A case of malignant melanoma arising from the oral cavity was successfully treated in Kurume University Hospital. This paper presents a report of this case.

\section{Case Report}

A 57-year-old female noticed tumor formation of the bilateral neck on November 10,1978 . The left cervical tumor increased in size gradually while the right cervical tumor did not present changes in size. Pain and tenderness were not noticed. The patient visited another clinic on December 22. A tumor formation on the right upper gingiva was pointed out and biopsy was done from the tumor. However, histological diagnosis was not established. The patient was referred to Kurume University Hospital on January 17, 1979 and admitted on the 19 th. She did not complain of nasal obstruction, trismus and visual disturbance.

A tumor, $2 \mathrm{~cm} \times 1.5 \mathrm{~cm}$ in size, was observed on the right upper gingiva. It was partly pigmented and partly whitish coated. Pigmentations and erosions were observed on the hard palate. Bilateral cervical lymphnodes were palpable. The right neck mass was $3.0 \mathrm{~cm} \times 2.0 \mathrm{~cm}$ in size and elastic hard. No fixation to the surrounding tissue was palpated. The left neck mass at the upper cervical node was $5.5 \mathrm{~cm} \times 3.5$ $\mathrm{cm}$ in size, elastic soft and mobile.

Results of laboratory examinations were within normal range. $\mathrm{X}$-ray films of the nose and paranasal sinuses revealed no abnormalities. Biopsy revealed that the lesion was malignant melanoma.

Partial maxillectomy and right radical neck dissection were performed under general anesthesia on February 8. Histological examinations of surgical specimens, both the primary and neck, showed malignant melanoma. Two weeks after the surgery, tumor formation was palpated at the spinal accesorry chain of the cervical lumph node. The tumor was extirpated on March 
8. Histologically, it was not a metastasis but scar tissue. The tumor of the left cervical region became smaller after hospitalization and did not appear to be a metastatic tumor. No treatment was indicated to the left cervical mass.

The patient was discharged on May 15, 1979. On July 31, 1979, the left superior deep cervical node became palpable at a follow-up examination. The tumor was $10 \mathrm{~cm}$ $\times 7 \mathrm{~cm}$ in size and elastic soft. The patient was admitted again on August 3. Left radical neck dissection was done under general anesthesia on August 9. The tumor involved the internal jugular vein. The extirpated tumor was elastic soft and contained necrotic tissue. Metastasis of malignant melanoma was detected histologically. The post-operative course was uneventful and the patient was discharged on August 22, 1979.

The patient is currently alive 2 years and 10 months after the initial treatment with no evidence of recurrent tumor.

\section{Discussion}

Malignant melanoma in the head and neck region is a rare condition. In particular, malignant melanomas of the oral cavity are rare. Chaudhry et al. (1958) reported that the incidence of malignant melanoma arising in the oral cavity was estimated to be 0.4 to $1.4 \%$ of all melanomas. In Japan, the incidence of melanoma arising in the oral cavity is relatively high. Takagi et al. (1974) reported that melanoma arising in the oral cavity occupied $7.5 \%$ of all malignant melanomas and $35 \%$ of all mucosal melanoma in Japan. Intraoral melanomas are twice as frequent in males as in females (Batsakis 1979; Chaudhry et al. 1958). Chaudhry et al. (1958) reported 93 cases of malignant melanoma of the oral cavity. Of these 93 cases, 75 cases located on the upper jaw. Ulcer formation or bleeding in the oral cavity is the most frequent initial complaint (Batsakis 1979). But pre-existing or concurrent melanoses can be found in the oral cavity (Chaudhry et al. 1958). The tumor is usually soft, painless and hemorrhagic. Diagnosis by clinical inspection alone is difficult. Biopsy should be done if melanoses is observed in the oral cavity (Batsakis 1979).

Radiotherapy is not very effective to malignant melanoma. Wide local excision should be indicated for the primary lesion. Radical neck dissection should be done if clinically involved lymphnodes are palpable. Mundth et al. (1965) and Goldsmith et al. (1970) reported that prophylactic neck dissection should be done even if clinically involved lymphnodes were not palpable. Our case was successfully treated with partial maxillectomy and radical neck dissection. Combined chemotherapy and immunochemotherapy may contribute to improve the prognosis (Saito et al. 1977).

Prognosis of malgnant melanoma arising in the oral cavity is desperate. Five-year survival rate is estimated to be 5 to $15 \%$ (Allen 1967; Alexander 1954; Conley 1963). The majority of patients die from tumor within 2 years.

\section{References}

Allen, A.C. (1967). The skin 2 nd Ed. C.V. Mosby Co. St. Louis.

Allexander, F. W. (1954). Malignant melanoma of the nasal septum. Laryngoscope, 64, 123129.

BAstSAKIS, J. G. (1979). Tumors of the head and neck. 2 nd Edition. The Williams and Silkins Company. Baltimore.

Chaudhry, A.P., Hmmpel, A. and Gorlim, R. J. (1958). Primary malignant melanoma of oral cavity. Review of 105 cases. Cancer, 11, 923-928.

Conley, J. J. and PAck, G.T. (1963). Melanoma of head and neck. Surg. Gynecol. Obstet. 116, 15-28.

Conley, J.J. and PACK, G.T. (1974). Melanomas of the mucous membranes of the head 
and neck. Arch. Otolaryngol, 99, 315-319.

Goldsmith, H. S., Shah, J. P. and KIM, D. H. (1970). Prognostic significance of lymph node dissection in treatment of malignant melanoma. Cancer, 26, 606-609.

Mundth, E. D., Guralnick, E. A. and Raker, J. W. (1965). Malignant melanoma clinical study of 427 cases. Ann Surg. 162, 15-28.
Saito, H., Tozawa, J., Kamota, M. and KasaHARA, M. (1977). Malignant melanoma of the nose and paranasal sinus. J. Otolaryngol. Jpn. 80, 1338-1351.

Takagi, M., Ichikawa, G. and Mori, W. (1974). Primary malignant melanoma of the oral cavity in Japan with special reference to mucosal melanomas. Cancer, 34, 358-370. 